\title{
STRUMA OVARII: TUMOR DE OVARIO INFRECUENTE
}

\section{Struma ovarii: infrequent ovarian tumor.}

\author{
Lilian S. Choc C. ${ }^{1}$, Miriam A. Choc C. ${ }^{2}$
}

\section{RESUMEN}

El estruma ovárico, neoplasia ovárica infrecuente, constituye un teratoma monodérmico altamente diferenciado hacia tejido tiroideo, cuyo diagnóstico certero lo proporciona la histopatología, siendo su tratamiento definitivo la cirugía.

Se presenta el caso de una paciente de $5 \mathrm{I}$ años de edad atendida en la 2 C Clínica Médica ALOM por dolor abdominal y tumoración de $8 \mathrm{~cm}$ en región pélvica derecha, corroborando por ultrasonido una masa anexial de ecogenicidad mixta en dicha región; con marcadores tumorales normales. La conducta quirúrgica consistió en histerectomía total con anexectomía bilateral, evidenciando el diagnóstico anatomopatológico de estruma ovárico del anexo izquierdo y teratoma quístico maduro en el anexo derecho.

Por consiguiente, los datos clínicos, los exámenes imagenológicos y anatomopatológicos permiten diagnosticar el estruma ovárico.

\section{ABSTRACT}

The Struma Ovarii, infrequent ovarian neoplasm, it constitutes a highly monodermic teratoma differentiated to the thyroid tissue, whose accurate diagnosis what provides the histopathology being the surgery the definitive treatment.

It presents the case of a patient of $5 \mathrm{I}$ years old attended in 2C Clínica Médica ALOM by abdominal pain and tumoration of 8 centimeters in right pelvic region, corroborating by ultrasound a right adnexal mass of mixed echogenicity in that region; normal levels of tumor markers. It decided the surgical intervention, it performed total hysterectomy with bilateral adnexectomy, evidencing the anatomopathological diagnosis of Struma Ovarii of the left annex and cystic teratoma mature in the right annex.

Therefore, the clinical data, the imaging and anatomopathological examinations allow to diagnose the ovarian struma.

\section{INTRODUCCIÓN}

El Estruma Ovárico (EO) es un Teratoma

EOvárico (TO) de la variante monodérmica, altamente especializado y de comportamiento benigno que ocasionalmente en un $5-10 \%$ de los casos, puede sufrir una transformación maligna. Las primeras descripciones fueron realizadas por Bottlin en 1889 y Von Kalden en 1895, siendo Ludwing Pick en 1903 quien identificó su característica fundamental, su constitución por tejido tiroideo ${ }^{1,2}$.

El EO es un tumor poco frecuente, que constituye el 2,5-2,7\% de los teratomas ováricos y alrededor del $0,3 \%$ de todas las neoplasias ováricas. El $20 \%$ de los teratomas ováricos contienen tejido tiroideo y el $5 \%$ de éstos son considerados EO, cuyo tejido tiroideo constituye más del $50 \%$ del tumor o que contiene menos de un $15 \%$ de tejido tiroideo pero altamente funcional o que presenta algún componente maligno derivado del tejido glandular tiroideo'.

Se presentan habitualmente entre la quinta y sexta década de la vida', si bien puede permanecer asintomático, el dolor pélvico es el síntoma más frecuente seguido de una masa pélvica generalmente unilateral, asociada a veces a síntomas de hipertiroidismo ${ }^{3}$.

La baja frecuencia de este tipo de TO nos obliga a individualizar una conducta terapéutica adecuada. Existe consenso en que el tipo de proceder quirúrgico a realizar en el EO depende del diagnóstico anatomopatológico intraoperatorio y de ser este benigno, la cirugía conserva-
Cirujana General-Videoasistida. Departamento de Cirugía General y Videoasistida. 2 C Clínica Médica ALOM

${ }^{2}$ Médico Anestesióloga. Departamento de Anestesiología. $2 \mathrm{C}$ Clínica Médica ALOM

Correspondencia a:

Dra. Lilian Soledad Choc Cac. Correo: dralchoc@hotmail.com Telf. y celular: 50250087688 y 50246563359

Palabras clave: Estruma de ovario, teratoma, neoplasias ováricas, ovario.

Keywords: Struma ovarii, teratoma, ovarian neoplasms, ovary.

Procedencia y arbitraje: no comisionado, sometido a arbitraje externo.

Recibido para publicación: 9 de mayo de 2018 Aceptado para publicación: 28 de diciembre de 2018

Citar como: Rev Cient Cienc Med 2018; 21(2): 51-55 
dora preserva la fertilidad futura en una mujer nulípara o en edad reproductiva con paridad aún insatisfecha, pudiendo realizarse quistectomías, Salpingooforectomía (SO) o la Histerectomía Abdominal Total (HAT) con salpingooforectomía bilateral (SOB) en pacientes con paridad satisfecha o cirugía radical en los casos infrecuentes de transformación maligna ${ }^{2,4}$.

Dada la poca frecuencia del tumor y los rasgos histopatológicos que lo caracterizan, se presenta un caso tratado en el Servicio de Cirugía General y Videoasistida de la $2 C$ Clínica Médica ALOM (2C CMA) de Santa Cruz Verapaz (SCV), Alta Verapaz (AV) Guatemala, C.A.

\section{PRESENTACIÓN DEL CASO}

Paciente de 51 años de edad que en fase perimenopaúsica consulta en octubre de 2017 en la 2C CMA ubicada en SCV AV por presentar dolor abdominal y una masa pélvica. Presenta antecedentes patológicos de artralgia generalizada, cefalea, ansiedad, hipertensión arterial tratada con irbesartan, lesión subepicárdica extensa tratada con Cardioaspirina ${ }^{\circledR}$, clopidogrel y ranolazine, además de hipercolesterolemia tratada con atorvastatina. Refiere una historia obstétrica de 11 partos eutócicos y esterilización quirúrgica por paridad satisfecha.

Al examen físico, se constató discreto dolor en abdomen inferior derecho, palpándose una masa en esa región. En el examen ginecológico, se visualizó un rectocistocele grado I y a la exploración vaginal, una tumoración anexial derecha de aproximadamente $8 \mathrm{~cm}$, dolorosa a la movilización.

La ultrasonografía ginecológica describe un útero en anteversión, aumentado de tamaño y de ecogenicidad normal que mide 4,26 x 8,06 x $3,77 \mathrm{~cm}$; una masa de ecogenicidad mixta anexial derecha que mide $6,95 \times 6,22 \times 6,97 \mathrm{~cm}$ sin calcificaciones y el Ovario Izquierdo (OI) de características normales que mide 2,29 × 1,89 cm. La citología exfoliativa vaginal resultó negativa para cáncer cervicouterino. Los marcadores tumorales CA-125 en $15,48 \mathrm{UI} / \mathrm{mL}$, HCG en 1,88 $\mathrm{UI} / \mathrm{mL}$ y AFP en $2,43 \mathrm{UI} / \mathrm{mL}$ dentro de límites normales.

Los complementarios iniciales, hemograma y hemoquímica resultaron normales, así como la radiología de tórax. El electrocardiograma constató una lesión subepicárdica anterior y por sugerencia del cardiólogo se realizó ecocardiograma de estrés con dobutamina, lo cual fue negativo para isquemia miocárdica. Las pruebas funcionales tiroideas TSH, T4 y T3 libres dentro de límites normales.

Se proporcionó plan educacional al paciente y familiares para salpingooforectomía derecha como tratamiento quirúrgico dada la masa anexial derecha existente y se firmó consentimiento informado necesario para realizar HAT con SOB, previa evaluación y autorización cardiológica preoperatoria. Los hallazgos intraoperatorios condicionaron la realización de una HAT con SOB.

La evaluación anatomopatológica de la pieza quirúrgica describió un útero piriforme de $8,4 \times 6,2 \times 3,0 \mathrm{~cm}$ con cérvix, cavidad intrauterina y oviductos sin lesiones macroscópicas evidentes. El OD midió $9,3 \times 7,5 \times 6,1 \mathrm{~cm}$, quístico con abundante tejido sebáceo y presencia de pelos (ver Fig. 1) y el Ol 4,0×3,7x1,8 cm, multiquístico contendiendo abundante tejido sebáceo e igualmente presencia de pelos (ver Fig. 2). A nivel microscópico, en el Ol se observó además, tejido compuesto por acinos de varios tamaños

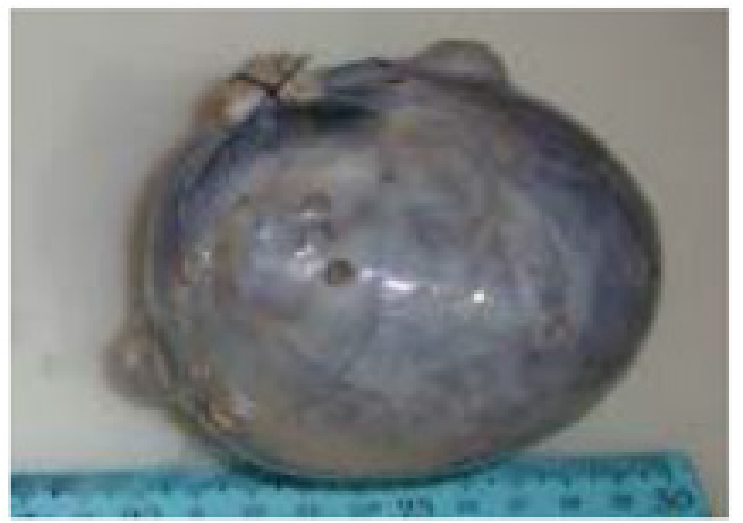

Figura 1: Ovario derecho que mide $9,3 \times 7,5 \times 6,1 \mathrm{~cm}$; quístico, de color grisáceo con abundante y fina vascularización. Al corte, de consistencia firme y abundante tejido sebáceo y la presencia de pelos.

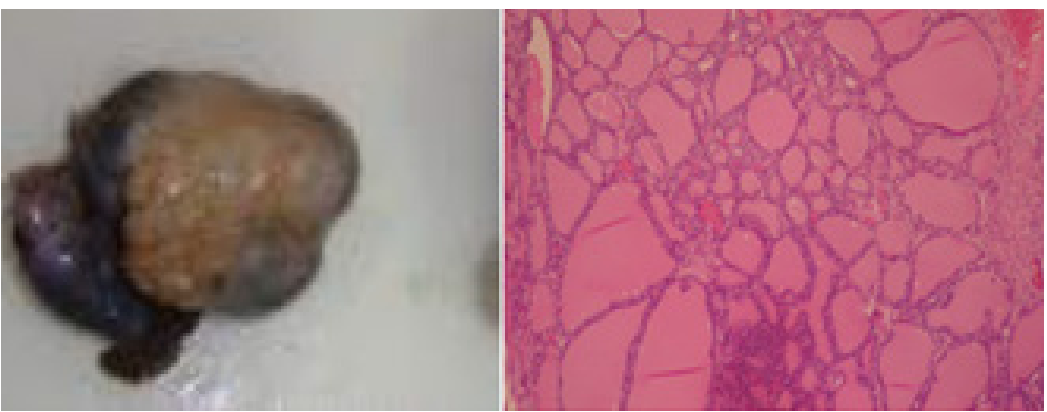

Figura 2: Ovario izquierdo que mide 4,0 × 3,7 × 1,8 cm, multiquístico de color grisáceo. Al corte, con abundante tejido sebáceo y la presencia de pelos. Los cortes histológicos corresponden a tejido tiroideo maduro que corresponde a Struma Ovarii. 
con material amorfo eosinofílico coloidal revestidos por una sola capa de epitelio columnar que corresponde histológicamente a tejido tiroideo maduro (ver Fig. 2). El diagnóstico anatomopatológico fue de un teratoma quístico maduro a nivel del OD e hidátide de Morgagni a nivel de la trompa uterina ipsilateral y estruma ovárico izquierdo, es decir, un teratoma monodérmico con tejido altamente diferenciado hacia tejido tiroideo.

La evolución postoperatoria de la paciente fue satisfactoria con calidad de vida aceptable. Se le proporcionó plan educacional para su seguimiento con Tiroglobulina (Tg), TSH, T3 y T4 libre y calcio sérico.

Se solicitó autorización a la paciente para publicar su caso y se le garantizó la confidencialidad de la información para proteger sus derechos.

\section{DISCUSIÓN}

Los tumores de ovario tienen una gran diversidad histológica y no siempre pueden ser clasificados según el diagnóstico citopatológico, afortunadamente en los teratomas el $20 \%$ son de origen germinal como es el caso del $\mathrm{TO}^{4}$. EI EO es un teratoma altamente especializado cuya baja frecuencia entre el 2,5-2,7\% constituye una de sus principales características además de la presencia de tejido tiroideo como característica fundamental de criterio anatomopatológico'.

Su comportamiento suele ser benigno aunque se describen características de malignidad en un $5-10 \%$ de los casos según diversos autores $^{3}$. El EO ha sido descrito con diseminación extra ovárica e histología benigna, en ausencia de un carcinoma somático de tipo tiroideo identificable que se denomina estrumosis peritoneal ${ }^{4}$.

La edad de presentación más frecuente es entre la quinta y sexta década de la vida ${ }^{1,5}$ aunque en la literatura se han descrito casos aislados en otras décadas de la vida ${ }^{4,6,7}$.

Clínicamente se manifiesta como masa tumoral abdominal cuyo tamaño puede oscilar desde $5-8 \mathrm{~cm}^{2}$ hasta $25 \mathrm{~cm}^{2}$ acompañado de dolor abdominal, pero en su mayoría son asintomáticos ${ }^{4}$.

Generalmente unilaterales, en el 6-15\% de los casos son teratomas bilaterales ${ }^{3,4}$. Se pueden presentar síntomas de hipertiroidismo en un $8 \%$ de los casos, siendo más frecuente si el tamaño del teratoma es superior a $3 \mathrm{~cm}$ describiéndose en un $17 \%$ asociados a ascitis y en un 5-6\% metástasis secundarias a EO maligno ${ }^{3}$.

Se han descrito tiroiditis inespecífica, tiroiditis de Hashimoto, linfoma primario, enfermedad de Graves, hiperplasia multinodular, bocio nodular y difuso y carcinoma papilar o folicular como formas de presentación ${ }^{6}$.

Es frecuente su asociación con cistoadenoma mucinoso, tumor de Brenner, tumor carcinoide, cistoadenoma seroso y quiste dermoide ${ }^{1,3,8}$. Como en nuestro caso que se relacionó con un quiste dermoide a nivel del anexo derecho.

La malignidad es diagnosticada según la atipia celular, actividad mitótica, invasión sanguínea o vascular y la presencia de metástasis, siendo los criterios más fiables la presencia de invasión vascular y la metástasis a distancia, pues la invasión capsular es difícil de establecer $^{6,8}$.

El diagnóstico diferencial se establece con el carcinoma de células claras y carcinoma endometrioide, cistoadenoma mucinoso, luteoma y el tumor de células de Sertoli-Leydig 1,3,4.

Rara vez se hace el diagnóstico preoperatorio de EO pues es difícil distinguir entre el EO y el quiste dermoide por las características imagenológicas ${ }^{1,3,9}$; si bien las técnicas diagnósticas por imagen son inespecíficas, la ecografía doppler es un método diagnóstico muy eficaz describiéndose un patrón de vascularización característico, que consiste en la existencia de un flujo sanguíneo alrededor de una cápsula que llega incluso hasta un área central, distribución dada por la alta vascularización del tejido tiroideo contrariamente al quiste dermoide por su contenido avascular ${ }^{1,3,9}$.

Asimismo, la tomografía, la resonancia magnética y la captación pélvica con lodo ${ }^{131}$ también han sido utilizados en el diagnóstico y orientación quirúrgica preoperatoria de estas pacientes ${ }^{4,10}$.

El tratamiento quirúrgico de elección en caso de benignidad es la quistectomía y la SO según deseos de paridad futura de la paciente, estando indicada la cirugía radical en caso de EO maligno $^{4,9}$.

El tipo de proceder quirúrgico a realizar, conservador o radical, en una paciente con EO debe ser individualizado de acuerdo al diagnóstico histopatológico intraoperatorio sea benigno o maligno y la paridad satisfecha de la mujer o no $0^{1,4,11}$. 
El estudio anatomopatológico del EO se establece por la presencia de tejido tiroideo de coloración marronácea con uno o varios quistes a nivel macroscópico y microscópicamente folículos tiroideos no encapsulados recubiertos por un epitelio cúbico reactivos a las técnicas de inmunohistoquímicas y ausencia de células C parafoliculares por su diferente origen embriológico, demostrándose gracias a las técnicas de inmunohistoquímica la presencia de $\mathrm{Tg}$ y $T 3^{6,12}$

Se recomienda realizar en el seguimiento postoperatorio, la determinación de Tg sérica a pesar del bajo poder metastásico de estos tumores. En caso de malignidad, se debe realizar tiroidectomía y rastreo corporal con lodo ${ }^{131}$ para localización de posibles metástasis y su ablación ${ }^{1,7}$.

\section{Conclusión}

Dada la variedad de diagnósticos a evaluar en una paciente con una masa pélvica, se reafirma la relevancia de una adecuada historia clínica y del empleo de técnicas imagenológicas, anatomopatológicas e inmunohistoquímicas para el diagnóstico certero y tratamiento quirúrgico individualizado de estruma ovárico.

\section{Agradecimientos}

Agradecemos al médico patólogo, Dr. Francisco Batres, personal del Laboratorio de Patología "El Imperio" de Cobán, Alta Verapaz, Guatemala, C.A., por el diagnóstico anatomopatológico definitivo, de Struma Ovarii de nuestra paciente.

\section{REFERENCIAS}

1. Barrios L, Ruiz K, Mendoza L, Gómez J. Estruma ovárico. Presentación de un caso y revisión de la literatura; Rev Colomb Obstet Ginecol [Internet]. 2014[Citado el 21 de abril 2018]; 65(1):79-84. Disponible en: http:// www.redalyc.org/html/1952/195231203009/.

2. Corrales Y, Ayala Z. Ovarian Teratoma. A Case Presentation. Revista Electrónica de las Ciencias Médicas en Cienfuegos[Internet]. 2012 [Citado el 21 de abril 2018]; 10(2):6672. Disponible en: http://www.redalyc.org/ pdf/1800/180023367011.pdf.

3. Bueno MM, García C, Pastor M, Lara R, Urbano MI. Struma ovarii. Propósito de un caso; Ginecología y Obstetricia del Hospital General Universitario Santa Lucía, Cartagena. XXVII Reunión Sociedad Ginecológica Murciana, 2017.

4. Pol M, Segundo O, López MV, Montejo A, González Y. Estruma ovárico. Presentación de Caso. Rev Cuban Endocrinol[Internet]. 2016[Citado el 21 de abril2018]; 27(2):156-62. Disponible en: http://www.revendocrinologia. sld.cu/index.php/endocrinologia/article/ view/33.

5. Rojo J, Anda E, Menéndez E, Echegoyen A, Martínez P. Hallazgo incidental de estruma ovárico. Endocrinol Nutr [Internet]. 2013[Citado el 24 de agosto 2018];60(5):268-
70. Disponible en: http://www.elsevier.es/ es-revista-endocrinologia-nutricion-12articulo-hallazgo-incidental-estruma-ovaricoS157509221200174X.

6. García CA, Sandoval DK, García E. Struma ovarii, hallazgos de patología tiroidea en el ovario. Rev Cuban Obstet Ginecol [Internet]. 2014[Citado el 21 de abril 2018];40(2). Disponible en: http://www.bvs.sld.cu/revistas/ gin/vol40_2_14/gin08214.htm.

7. Guanipa $\bar{W}$, FernándezP, Álvarez M, Figueroa R. Struma ovarii con histología maligna: un caso con carcinoma papilar de tiroides. Endocrinol Nutr [Internet]. 2011[Citado el 21 de mayo 2018];58(1):48-50. Disponible en: $h t t p: / /$ www.elsevier.es/es-revista-endocrinologianutricion-12-articulo-struma-ovarii-conhistologia-maligna-S1575092210002846.

8. Parrilla ME, López MV, Valls O: Ovario. En: Parrilla ME, López MV, Valls O. Atlas de ecocitopatología diagnóstica en las lesiones abdominales. Habana, Cuba: Editorial Ciencias Médicas de Habana; 2006. p.261-344.

9. Alvarado A, Tejeira E. Teratoma de ovario; Rev Méd Cient[Internet]. 2012[Citado el 21 de abril 2018];25(1):46-7. Disponible en: http:// www.revistamedicocientifica.org/index.php/ rmc/article/viewFile/326/pdf_35.

10.Shen J, Xia X, Lin Y, Zhu W, Yuan 
J. Diagnosis of Struma ovarii with medical imaging. Abdom Imaging [Internet].2011[Citado el 21 de abril 2018];36(5):627-31.Disponible en: https:// www.ncbi.nlm.nih.gov/pubmed/21104246.

11. Labiano T, Echeveste JI, Idoate MA, Bondía JM, Lozano MD. Struma ovarii maligno no sospechado: diagnóstico citológico mediante punción aspirativa con aguja fina de lesión vertebral metastásica. Rev Esp de Patol[Internet].2012[Citado el 21 de abril 2018];45(4):238-42. Disponible en: http:// www.elsevier.es/en-revista-revista-espanolapatologia-297-articulo-struma-ovariimaligno-no-sospechado-S1699885512000487.

12. Arab C, Cisterna P, Farías L, Escalona J, Cornejo V, Moreno M, et al. Struma carcinoide de ovario: Reporte de dos casos. Rev Obstet Ginecol[Internet]. 2008[Citado el 11 de mayo 2018];3(3):199-202.Disponible en: http://www.revistaobgin.cl/articulos/ver/495. 